\title{
A unique bZIP transcription factor imparting multiple stress tolerance in Rice
}

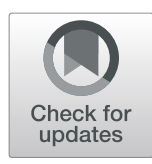

\author{
Priyanka Das ${ }^{1}$, Nita Lakra', Kamlesh Kant Nutan ${ }^{1}$, Sneh Lata Singla-Pareek ${ }^{2}$ and Ashwani Pareek ${ }^{1 *}$ D
}

\begin{abstract}
Background: Rice productivity is adversely affected by environmental stresses. Transcription factors (TFs), as the regulators of gene expression, are the key players contributing to stress tolerance and crop yield. Histone gene binding protein-1b (OsHBP1b) is a TF localized within the Saltol QTL in rice. Recently, we have reported the characterization of OsHBP1b in relation to salinity and drought tolerance in a model system tobacco. In the present study, we over-express the full-length gene encoding OsHBP1b in the homologous system (rice) to assess its contribution towards multiple stress tolerance and grain yield.

Results: We provide evidence to show that transgenic rice plants over-expressing OsHBP1b exhibit better survival and favourable osmotic parameters under salinity stress than the wild type counterparts. These transgenic plants restricted reactive oxygen species accumulation by exhibiting high antioxidant enzyme activity (ascorbate peroxidase and superoxide dismutase), under salinity conditions. Additionally, these transgenic plants maintained the chlorophyll concentration, organellar structure, photosynthesis and expression of photosynthesis and stressrelated genes even when subjected to salinity stress. Experiments conducted for other abiotic stresses such as drought and high temperature revealed improved tolerance in these transgenic plants with better root and shoot growth, better photosynthetic parameters, and enhanced antioxidant enzyme activity, in comparison with WT. Further, the roots of transgenic lines showed large cortical cells and accumulated a good amount of callose, unlike the WT roots, thus enabling them to penetrate hard soil and prevent the entry of harmful ions in the cell.

Conclusion: Collectively, our results show that rice HBP1b gene contributes to multiple abiotic stress tolerance through several molecular and physiological pathways and hence, may serve as an important gene for providing multiple stress tolerance and improving crop yield in rice.
\end{abstract}

Keywords: ABA, bZIP transcription factor, Callose, Oryza sativa, Photosynthesis, Salinity, Heat

\section{Background}

Soil salinity, soil dehydration, and extreme environmental temperatures significantly influence plant growth and development and finally result in compromised crop yield (Cramer et al. 2011). The molecular response of plants towards these stresses has been reported to be highly complex; an interplay of many genes involved in perception and signal transduction (Pareek et al. 2010). Among these genes, the transcription factors (TFs) have been reported as key players in determining the survival and yield of plants under stress conditions (Lakra et al. 2015; Nutan et

\footnotetext{
* Correspondence: ashwanip@mail.jnu.ac.in

${ }^{1}$ Stress Physiology and Molecular Biology Laboratory, School of Life Sciences, Jawaharlal Nehru University, New Delhi 110067, India

Full list of author information is available at the end of the article
}

al. 2017). bZIP (basic leucine zipper) group is one of the major group of transcription factors which has expanded and evolved in genomes to play a crucial role in plant growth and stress response. bZIP proteins are a class of unique TFs with the bZIP domain and two structural features: a leucine zipper domain and a basic DNA binding domain (Nijhawan et al. 2008). bZIP proteins are evidently seen throughout the plant kingdom participating in various physiological processes such as seed germination, flower development and fertility, plant senescence, abiotic stress responses and ABA signal transduction (Lee et al. 2006; Nijhawan et al. 2008; Zou et al. 2008; Alves et al. 2013). Although, transgenic plants overexpressing the bZIP proteins have been reported to exhibit a higher tolerance to abiotic stresses such as 
salinity, drought and extreme temperature conditions (Lee et al. 2006; Zhang et al. 2008; Liao et al. 2008; Liu et al. 2014; Lakra et al. 2015), the physiological and molecular basis of tolerance in these plants largely remains unexplored. Furthermore, the role of bZIP proteins towards maintaining the yield in crop plants under stress conditions is yet to be reported.

Rice (Oryza sativa L.) is a major crop plant and staple food for half of the world's population (Pareek et al. 2010). But, the fact is that most of the high yielding and popular varieties of rice such as IR64 are sensitive to abiotic stresses. Hence, there is an urgent need to understand the response of this crop towards stresses, especially salinity and drought which are severely affecting the crop yield (Pareek et al. 2010). Through gene expression analysis in salinity tolerant genotypes of rice, it has been clearly established that array of genes are constitutively expressed at a higher level to make the plant salinity tolerant (Kumari et al. 2009; Nutan et al. 2017; Soda et al. 2013). Salinity stress has been documented to induce a set of Saltol QTL-localized genes including Histone gene binding protein/transcription factor-1b (HBP1b) which is also differentially expressed among the contrasting genotypes of rice (Kumari et al. 2009).

HBP1b is categorized under bZIP family. According to the structure and DNA binding specificity of the molecule, HBP TFs are divided into two major groups named as; HBP1a and HBP1b (Jakoby et al. 2002). HBP1a are also known as GBFs (G-box binding factors) and are well characterized as they preferentially bind to 'CACGTG' and 'ACGTCA' sequences. The other conserved group is composed of $H B P 1 b$ which bind to TGACG (T/C) sequences and have a bZIP domain close to the N-terminus. Tabata et al. (1991) have shown the binding of HBP1b TFs to the histone (H3) gene promoter and many reports have revealed that the plant histone genes may play key roles in diverse stress responses (Tabata et al. 1991; Schultz et al. 1996; Bray et al. 1999). As it is clear that the transcription factor HBP1b in rice is salinity inducible (Kumari et al. 2009) and is localized within a QTL for salinity tolerance, we hypothesized that this gene could function as a key player for salinity tolerance in the rice plant. Ectopic expression of this $H B P 1 b$ gene in a heterologous system (tobacco) and analysis of stress tolerance by our group (Lakra et al. 2015) provided the clue that over-expression of this gene could affect the expression of several salinity responsive downstream genes. Additionally, it could also serve as a 'master gene' contributing to the improved growth and yield under abiotic stress conditions. To test this theory, we have now over-expressed the full-length HBP1b (Gene ID- LOC_Os01g17260) in the homologous system (O. sativa L. CV. IR64) and analysed the behaviour of the transgenic plants over-expressing rice $\mathrm{HBP} 1 \mathrm{~b}$ under different abiotic stress conditions, with special emphasis on the plant growth and yield under salinity. In the present work, we also dissect out molecular mechanism of stress tolerance in these plants as mediated through the altered expression of the key genes involved in photosynthesis and stressresponse.

\section{Results \\ Nuclear-localized bZIP transcription factor OsHBP1b exhibits developmental regulation and differential expression under diverse abiotic stresses}

In our previous study, we have shown that OsHBP1b is a bZIP type protein and is very much related to its orthologs from wheat and barley (Lakra et al. 2015). By GFP-fusion assay, it has also been confirmed that the OsHBP1b protein is localized in the nucleus (Lakra et al. 2015). It has also been reported that transcription factors express at specific stages of plant development to perform a specific role (Ramachandran et al. 1994). These observations prompted us to analyse the transcript abundance for the $O s H B P 1 b$ at different developmental stages of rice. For this purpose, expression levels of $O s H B P 1 b$ were analyzed in rice under different developmental stages through the publicly available microarray databases (https://www.genevestigator.com/gv/). Expression of OsHBP1b was checked at nine different growth stages of rice and the results are presented in Fig. 1. The analysis confirmed that OsHBP1b transcript is highly abundant in rice during all the developmental stages with its highest expression at the booting stage and lowest expression during the germination stage (Fig. 1a). Furthermore, OsHBP1b showed differential transcript abundance in various tissues, where, a medium to high expression was found in different tissue types (Fig. 1b). In comparison to other tissues, pollen tissue showed the lowest expression of OsHBP1b transcript (Fig. 1b). In confirmation with the microarray results (Fig. 1b), quantitative real- time PCR analysis showed a higher transcript expression for OsHBP1b in the leaf tissue followed by the root and the embryo (Fig. 1c).

In our previous study, we have shown that OsHBP1b is localized within the Saltol QTL and also inducible by salinity (Lakra et al. 2015). In order to check if this gene is responsive to diverse abiotic stresses in addition to salinity, we have performed expression analysis of $O s H B P 1 b$ under various stress conditions (Fig. 1e - h). We found that though there are no significant changes in the transcript abundance of this gene in rice seedlings under control conditions over a period of $24 \mathrm{~h}$ (Fig. 1d), the gene is inducible in response to diverse abiotic stresses such as; salinity (12 to 15 fold), high temperature (8 to 10 fold), drought (20 to 22 fold) and ABA (14 to 16 fold) (Fig. 1e - h). 


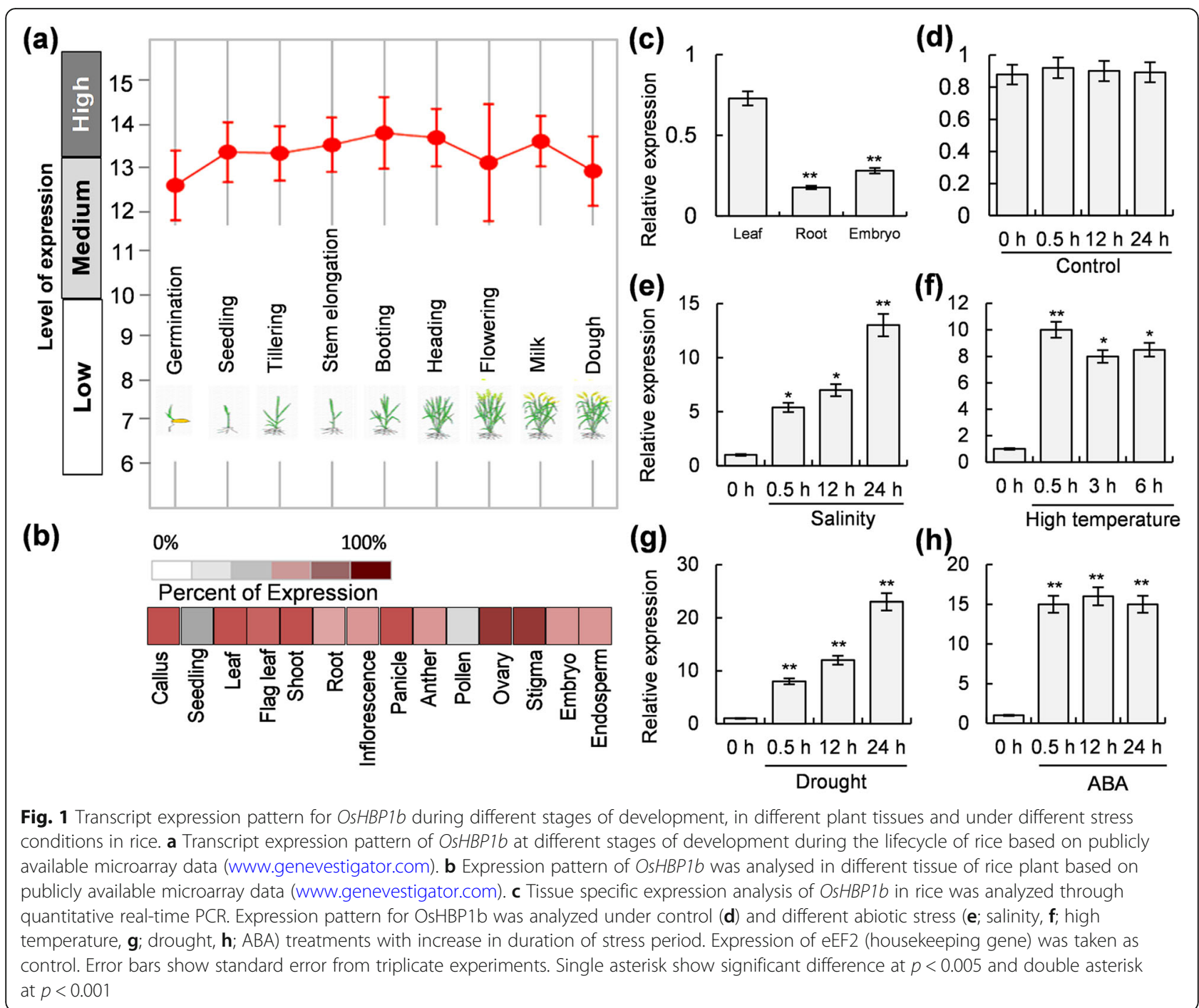

\section{Generating transgenic 0 . sativa cv IR64 over-expressing OsHBP1b}

To raise the transgenic rice, OsHBP $1 b$ was cloned into plant transformation vector pCAMBIA1304, using genespecific forward and reverse primer pairs having specific restriction sites (Additional file 5: Table S1). For rice transformation, a highly efficient and reproducible Agrobacterium tumefaciens-mediated rice transformation protocol has been followed which has been standardized and published by our group, where mature seeds are taken as explants (Sahoo et al. 2011). Growing callus was transformed with positive OsHBP1b-recombinant $A$. tumefaciens. Transgenic plants were selected on MS media having $50 \mathrm{mg} / \mathrm{L}$ hygromycin. The transgenic plants which developed proper root in hygromycincontaining MS media were screened by tissue PCR with a combination of gene-specific forward and vector specific reverse primer pair (Additional file 5: Table S1 and Additional file 1: Figure S1a). For negative and positive controls, genomic DNA from wild type rice (WT; nontransgenic) and pCAMBIA1304-OsHBP1B plasmid respectively were taken as a template. Out of 89 plants regenerated, 17 (eight lines are shown here) were found to be positive as they showed the desired amplicon from the genomic DNA (Additional file 1: Figure S1a). Southern blot analysis confirmed the presence of a single copy of the transgene in two lines viz. L2 and L7 (Additional file 1: Figure S1b) which were then used for further experiments. Quantitative real-time PCR and western blot analysis revalidated the transgenic nature of these two lines with 4-5 fold higher accumulation of the $O s H B P 1 b$ transcript and more expression of protein than the WT (Additional file 1: Figure S1c and Additional file 1: Figure S1d).

Transgenic rice plants over-expressing OsHBP1b tolerate high level of salinity and maintain chlorophyll and ROS levels To assess the relative tolerance of OsHBP $1 b$ overexpressing rice plants towards salinity stress, quick 
testing was carried out using leaf segments of WT and the transgenic lines (for ease, these lines are henceforth referred as L2 and L7 in the manuscript). Leaf segments kept in $\mathrm{NaCl}$ solution confirmed early bleaching in the WT tissues than L2 and L7 plants (Fig. 2a). It was visibly seen that the L2 and L7 leaf segments could stay green by retaining a higher level of chlorophyll up to $72 \mathrm{~h} \mathrm{NaCl}(300$ $\mathrm{mM}$ ) stress, unlike WT plants which showed very early bleaching (Fig. 2a). Under $\mathrm{NaCl}$ stress, the loss of chlorophyll in the WT plants was higher than L2 and L7 plants (Fig. 2b). It was observed that $300 \mathrm{mM} \mathrm{NaCl}$ treatment caused $\sim 80 \%$ decrease in total chlorophyll level in the WT as compared to the control plants, while, barely $35-40 \%$ decrease was observed for the OsHBP1b over-expressing plants.

We examined the status of superoxide $\left(\mathrm{O}_{2}{ }^{-}\right)$and hydrogen peroxide $\left(\mathrm{H}_{2} \mathrm{O}_{2}\right)$ in the WT, L2 and L7 transgenic lines through NBT and DAB staining, under control as well salinity-imposed condition $(200 \mathrm{mM} \mathrm{NaCl})$.
The accretion of $\mathrm{O}_{2}{ }^{-}$(Fig. 2c) and $\mathrm{H}_{2} \mathrm{O}_{2}$ (Fig. 2d) under control and salt stress was seen to be considerably less for the L2 and L7 than the WT plants. Spectrophotometric analysis for $\mathrm{H}_{2} \mathrm{O}_{2}$ measurement also revealed that the level of $\mathrm{H}_{2} \mathrm{O}_{2}$ in the $\mathrm{L} 2$ and $\mathrm{L} 7$ transgenic lines is lower $(\sim 33 \%)$ than the WT under $\mathrm{NaCl}$ stress. However, there was an insignificant difference in the level of $\mathrm{H}_{2} \mathrm{O}_{2}$ in WT and transgenic lines under control conditions (Fig. 2e).

\section{Transgenic rice plants over-expressing rice $H B P 1 b$ can tolerate high salinity by maintaining physiological and enzymatic homeostasis}

Seven days old $\mathrm{T}_{2}$ generation rice seedlings were transferred to hydroponic solution containing $200 \mathrm{mM} \mathrm{NaCl}$ for $3 \mathrm{~d}$. Morphological indicators were analysed firstly to check the salinity response of $O s H B P 1 b$ over-expressing lines. Although, there was a difference in shoot length of

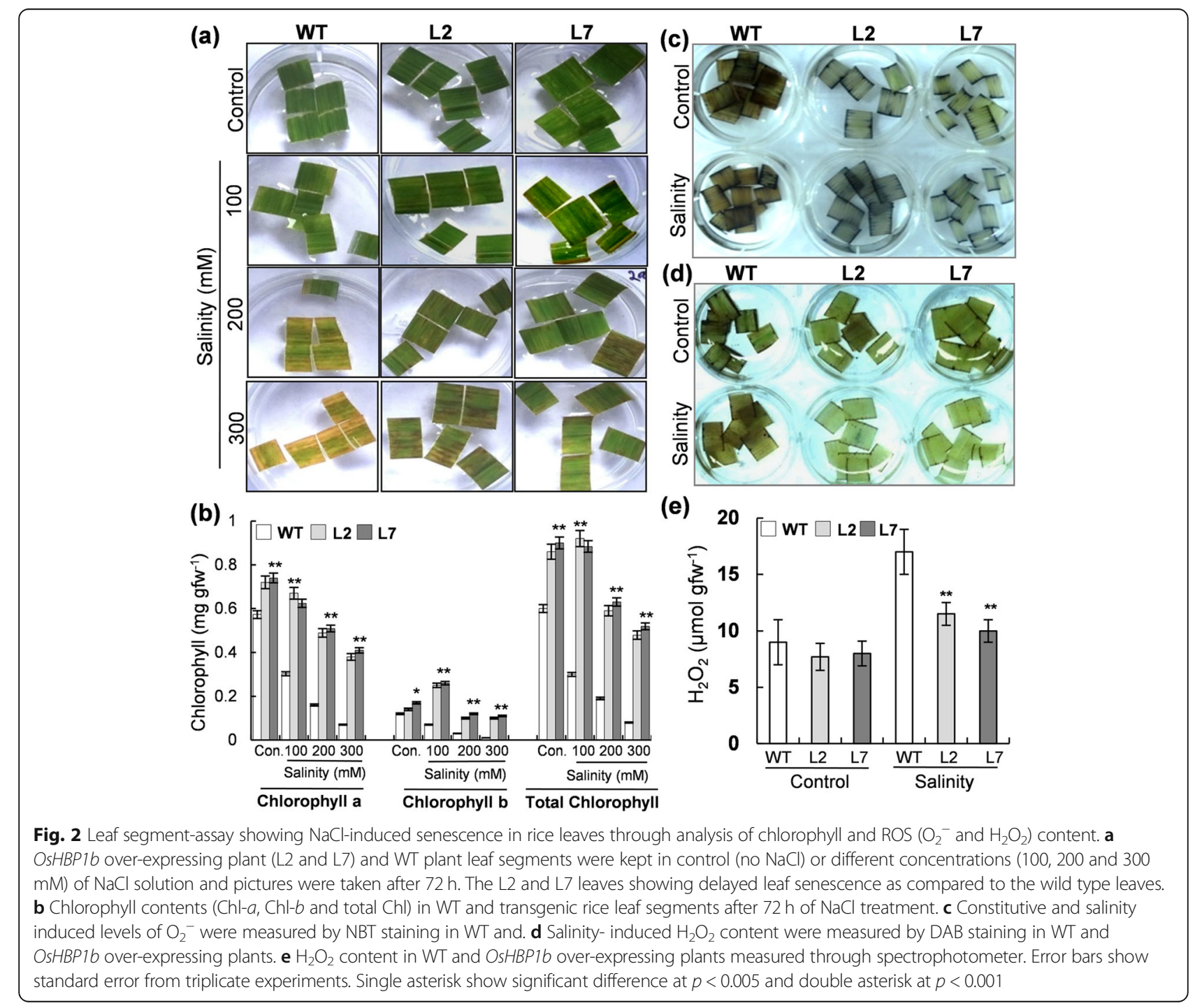


WT and OsHBP1b over-expressing seedlings, no significant changes were observed in their root length and root phenotype under control conditions (Fig. 3a). L2 and L7 transgenic lines showed a robust root system with more length and higher number of adventitious roots which grew thicker and longer than the wild type plants under salinity (Fig. 3b and d). Under salinity, shoot length and fresh weight of the L2 and L7 lines were also found to be increased than the WT plant (Fig. 3c and e). Electrolyte leakage (EL), a primary indicator of membrane damage under stress (Verslues et al. 2006), was measured in the $O s H B P 1 b$ over-expressing transgenic plants. Under control conditions, we could not get significant differences in the electrolyte leakage between WT and the transgenic lines (Fig. 3f). On the other hand, under salinity stress, the over-expressing lines showed a comparatively lower (50\%) EL than the WT plants (Fig. 3f). To check if OsHBP1b-mediated increase in salt stress tolerance resulted by the mitigation of the lethal $\mathrm{Na}^{+}$ions, the $\mathrm{Na}^{+}$and $\mathrm{K}^{+}$contents in the WT, L2 and L7 plants was measured. The $\mathrm{K}^{+} / \mathrm{Na}^{+}$ratio was found to be higher in the leaves of the transgenic plants than the wild type

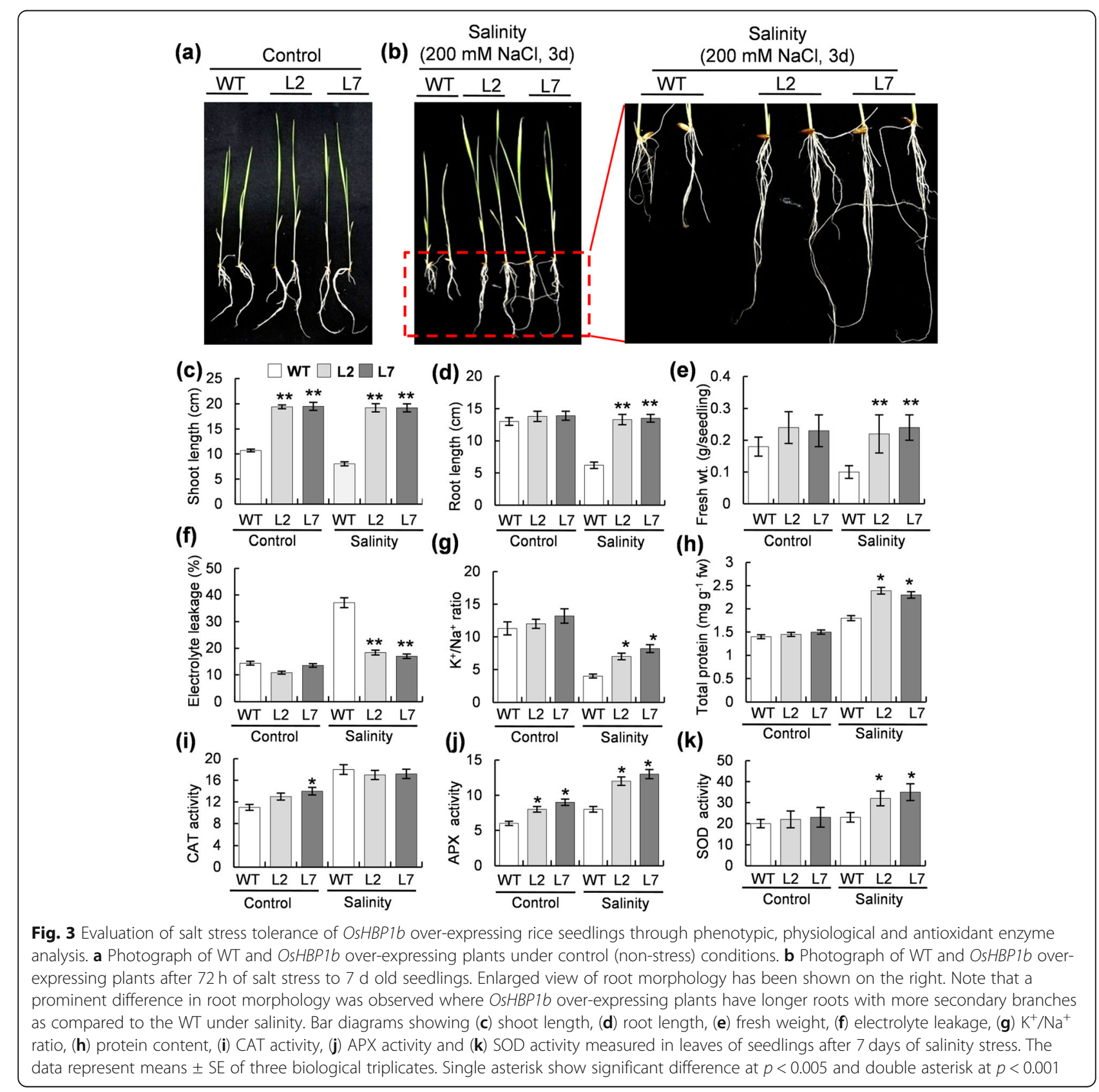


(Fig. 3g), whereas, no significant change in $\mathrm{K}^{+} / \mathrm{Na}^{+}$ratio was observed in WT and over-expressing lines under control conditions (Fig. 3g).

We measured the total protein level and carried out antioxidant enzyme activity assays in control as well as salinity-treated leaves from WT and OsHBP1b overexpressing seedlings. Under control conditions, WT, L2 and L7 plants showed a comparable level of protein, whereas, under salinity, L2 and L7 lines showed 20\% higher protein content than the WT plants (Fig. 3h). Though CAT activity was found to remain largely unchanged in transgenic lines and WT seedlings under salinity stress (Fig. 3i), increased APX $(\sim 43 \%)$ and increased SOD (20-30\%) activity was quite evident under similar conditions (Fig. $3 \mathbf{j}$ and $\mathrm{k}$ ). These results also indicate that the expression levels of OsHBP1b in rice are correlated with salinity tolerance in transgenic lines as mediated via maintenance of cellular physiology and enzymatic homeostasis.

\section{Stomatal functions and organelle structure contribute towards salinity tolerance in the OsHBP1b over- expressing plants}

The OsHBP1b over-expressing lines showed healthy cellular structure with intact chloroplasts and proper thylakoid stacking under salt stress, unlike the wild type plants. In wild type plants, severe cell damage and distorted chloroplast and thylakoid stacking was found when leaf sections from salinity treated plants were analyzed through TEM (Fig. 4a). In WT and transgenic lines, a similar type of stomatal phenotype was observed under control conditions, whereas under salinity stress, the stomata of L2 and L7 transgenic lines were found to be largely closed and protected (Fig. 4b). These findings suggest enhanced tolerance of the transgenic plants towards salinity stress as compared to the WT plants via maintenance of healthy cellular organelles and protected stomata.

\section{Rice plants over-expressing OsHBP1b exhibit efficient photosynthesis and set viable seeds under salinity} Phenotypic analysis revealed a better morphology of $O s H B P 1 b$ over-expressing plants under control as well as $200 \mathrm{mM}$ of salinity stress (Fig. 5a). The plant height for the transgenic lines was found to be higher than the WT under control as well as salinity conditions (Fig. 5a). Under non-stress conditions, both WT and transgenic plants grew well and set seeds upon maturity. However, under salinity, the WT plants could not grow well and failed to set flower even after recovery (under conditions of adequate water availability). On the other hand, the transgenic plants could flower normally and set seed following salinity treatment and subsequent recovery (Fig. 5a and b). Analysis of the root anatomy of WT and transgenic lines grown under control condition showed that the transgenic lines possess an enlarged stele and bigger cortical parenchyma cells with an expanded cortex (Fig. 5c, upper panel). Also, aniline blue staining revealed that the roots of transgenic lines accumulate a good amount of callose in the cortex, unlike the WT roots (Fig. 5c, lower panel).

Further, various photosynthesis related parameters for the WT and transgenic lines under control and salinity stress were studied using IRGA. Under control conditions, no significant variations were observed between WT and the transgenic lines in terms of their $\mathrm{Fv} / \mathrm{Fm}$ ratio, Electron transport rate (ETR), Non-photochemical quenching (NPQ) and $\mathrm{CO}_{2}$ assimilation (Fig. 5d-g). A drastic decrease in $\mathrm{Fv} / \mathrm{Fm}$ ( 32\%), ETR (50 to $65 \%$ ) and $\mathrm{CO}_{2}(\sim 70 \%)$ assimilation were observed in WT as compared to the transgenic lines under salinity conditions (Fig. 5d, e, and g). However, salinity did not had any significant adverse effect on the transgenic lines, in terms of their $\mathrm{Fv} / \mathrm{Fm}$ ratio, ETR and $\mathrm{CO}_{2}$ assimilation. As expected, the non-photochemical quenching (NPQ) in the WT plants increased under salinity as compared to the transgenic lines (Fig. 5f). Furthermore, the relative water content and the rate of transpiration was found to be higher ( 43 and $49 \%$ respectively) in the transgenic lines than the WT plants under salinity conditions, while there was no change observed between the WT and transgenic lines under normal growth conditions (Fig. 5h and i).

\section{The transgenic OSHBP1b over-expressing lines exhibit altered expression of photosynthesis- and stress-related genes}

To analyse the expression of photosynthesis- and stress-related genes, we performed quantitative realtime PCR for a few selected genes in rice seedlings under control and salinity conditions. Under control conditions, significantly higher levels of expression was observed for most of the selected photosynthesisrelated genes such as; rbcL $(\sim 1.3$ fold $)$, psbA $(\sim 8$ fold), LHCP2 (2.5 fold), CAO ( 1.7 fold) and POR ( 3.3 fold) in the OsHBP1b over-expressing plants as compared with the WT (Fig. 6a). Only psaA gene showed an insignificant difference in the expression pattern when compared to WT. Most of the selected genes in both types of plants showed decreased expression under salinity, but it was evident that even after salt treatment, the level of expression of all the selected photosynthesis-related genes in OsHBP1b over-expressing lines was comparable to the level of expression of the selected genes in the salt-untreated (control) WT plant (Fig. 6a).

Apart from photosynthesis-related genes, we also investigated the expression kinetics of various stress- 


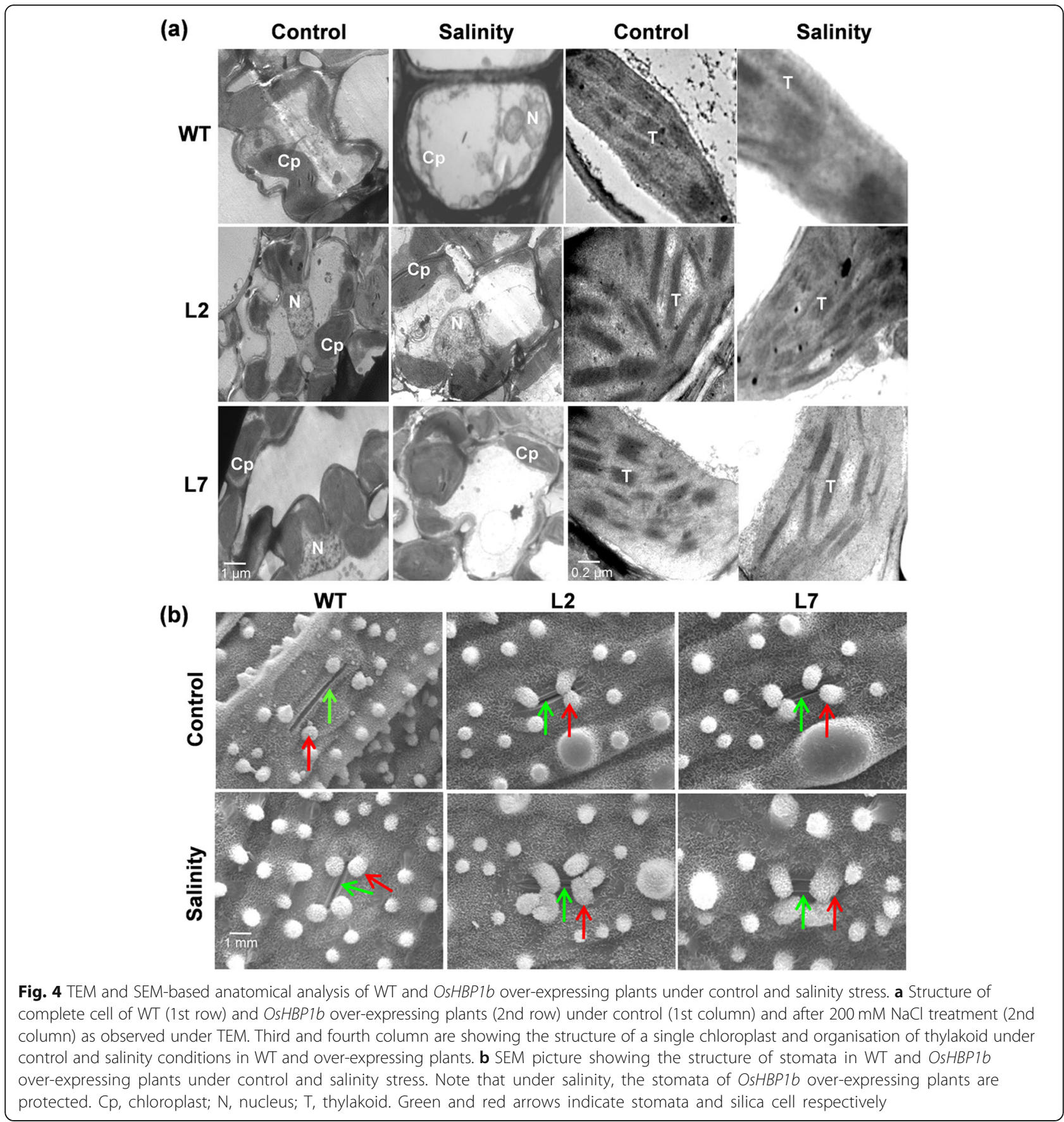

related genes in the OsHBP $1 b$ over-expressing lines. Under control conditions, there were three types of expression patterns observed viz. (i) most of the genes (ascorbate peroxidase; APX, NAC6 TF, DREB TF, Proline biosynthetic gene; P5CS2 and bZIP TF) showed increased expression in OsHBP1b over-expressing lines, (ii) one of the genes (SOD) showed decreased expression in $O s H B P 1 b$ over-expressing lines and (iii) for some of the other genes (catalase; CAT, heat shock TF; HSTF and heat shock protein; HSP), no change in expression was observed between WT and OsHBP1b overexpressing lines (Fig. 6b). However, after salinity treatment, there were higher levels of expression (SOD, $\sim 4$ fold; APX, $\sim 5$ fold; DREB, $\sim 7$ fold; NAC, $\sim 2.5$ fold, bZIP, 1.5 fold and HSP, $\sim 4$ fold) observed in OsHBP1b over-expressing lines, except CAT, P5CS2 and HSTF (Fig. 6b). A decreased (3.5 fold) level of CAT transcript was observed in the OsHBP1b over-expressing lines as compared with WT under salinity stress (Fig. 6b). No change in P5CS2 and HSTF transcripts were evident in 


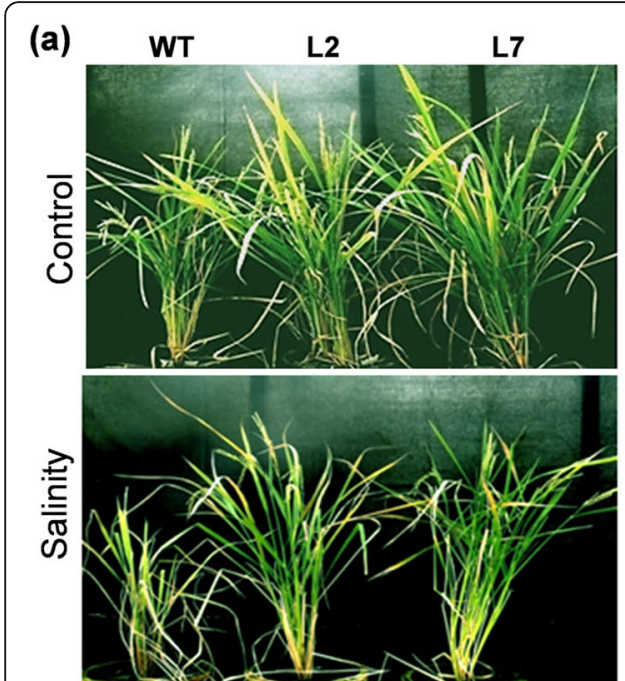

(d)
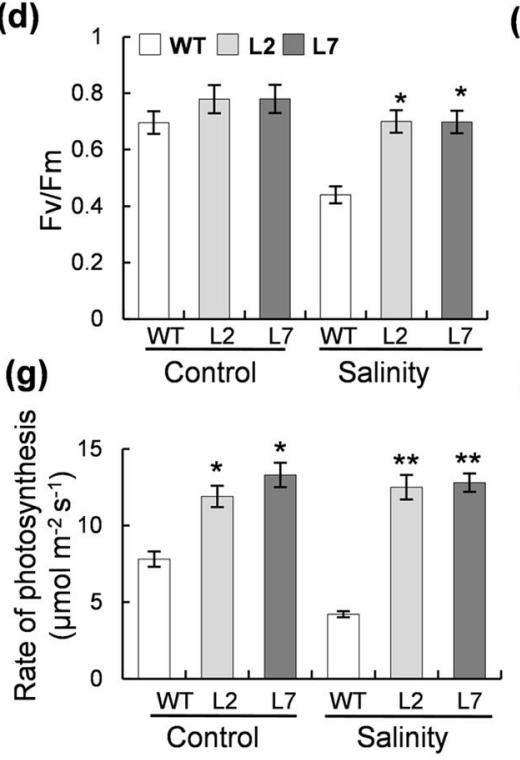

(e)
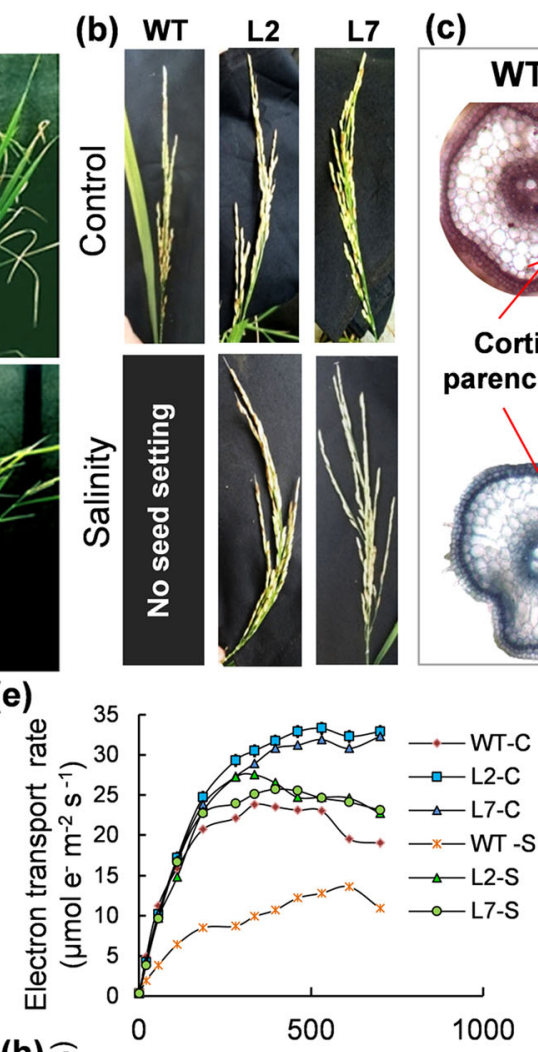

(h)

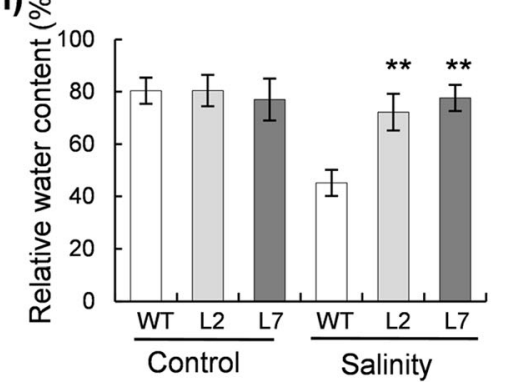

(c)

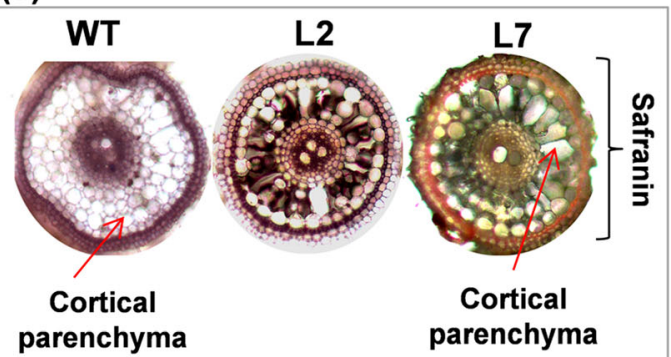

parenchyma

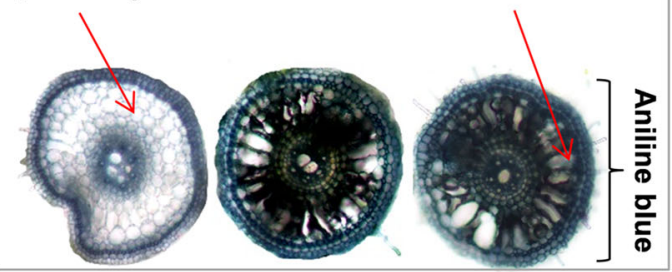

(f)

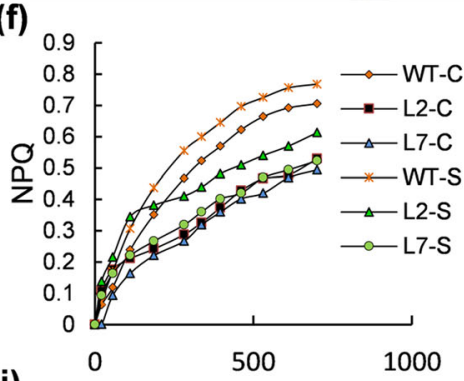

(i)

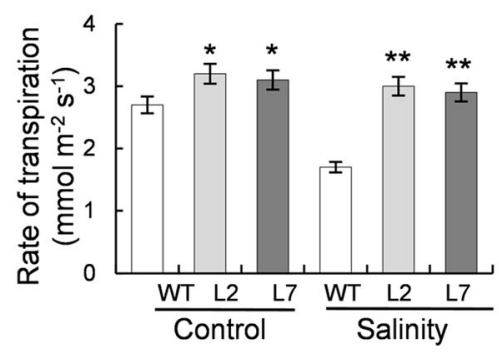

Fig. 5 Assessment of physiological and morphological parameters of mature OsHBP1b lines as compared to the WT under salinity. a Pictorial view of mature WT and OSHBP1b over-expressing plants. Note the better growth in terms of plant height, and number of leaves in the OsHBP1b over-expressing plants as compared to WT under both control and salinity conditions. b Panicle morphology of WT and OsHBP1b over-expressing plants. Note that under salinity, there was no panicle produced in the WT plants. c Anatomy of the root of WT and OsHBP1b over-expressing plants stained with safranin (upper panel) or aniline blue (lower panel) under control conditions. d Fv/Fm, (e) electron transport rate, (f) NPQ, (g) rate of photosynthesis, $(\mathbf{h})$ relative water content and (i) rate of transpiration, in OsHBP1b over-expressing plants as compared with the WT. Error bars show standard error from triplicate experiments. Single asterisk show significant difference at $p<0.005$ and double asterisk at $p<0.001$

OsHBP1b over-expressing lines as compared to the WT, under salinity (Fig. 6b).

\section{Over-expression of OsHBP1b in transgenic rice plants enable them to tolerate multiple abiotic stresses}

Apart from salinity, we have analysed the performance of $O s H B P 1 b$ over-expressing lines under diverse abiotic stresses by exposing the plants to drought, high temperature, and $\mathrm{ABA}$. It was evident that the following recovery after drought (PEG) and high-temperature treatment, the transgenic lines over-expressing OsHBP $1 b$ performed better having higher shoot length, root length and fresh weight (Additional file 2: Figure S2 a-c and Additional file 3: Figure S3 a-c). In both the stress treatments, the root morphology was found to be more favourable in the transgenic lines as compared to the WT where more number of secondary and adventitious roots were evident (Additional file 2: Figure S2a and Additional file 3: Figure S3a). In order to assess their photosynthesis capacity, we checked the $\mathrm{Fv} / \mathrm{Fm}$ in these plants and found a higher $\mathrm{Fv} / \mathrm{Fm}$ value in the OsHBP1b over-expressing lines as compared to the WT, under 


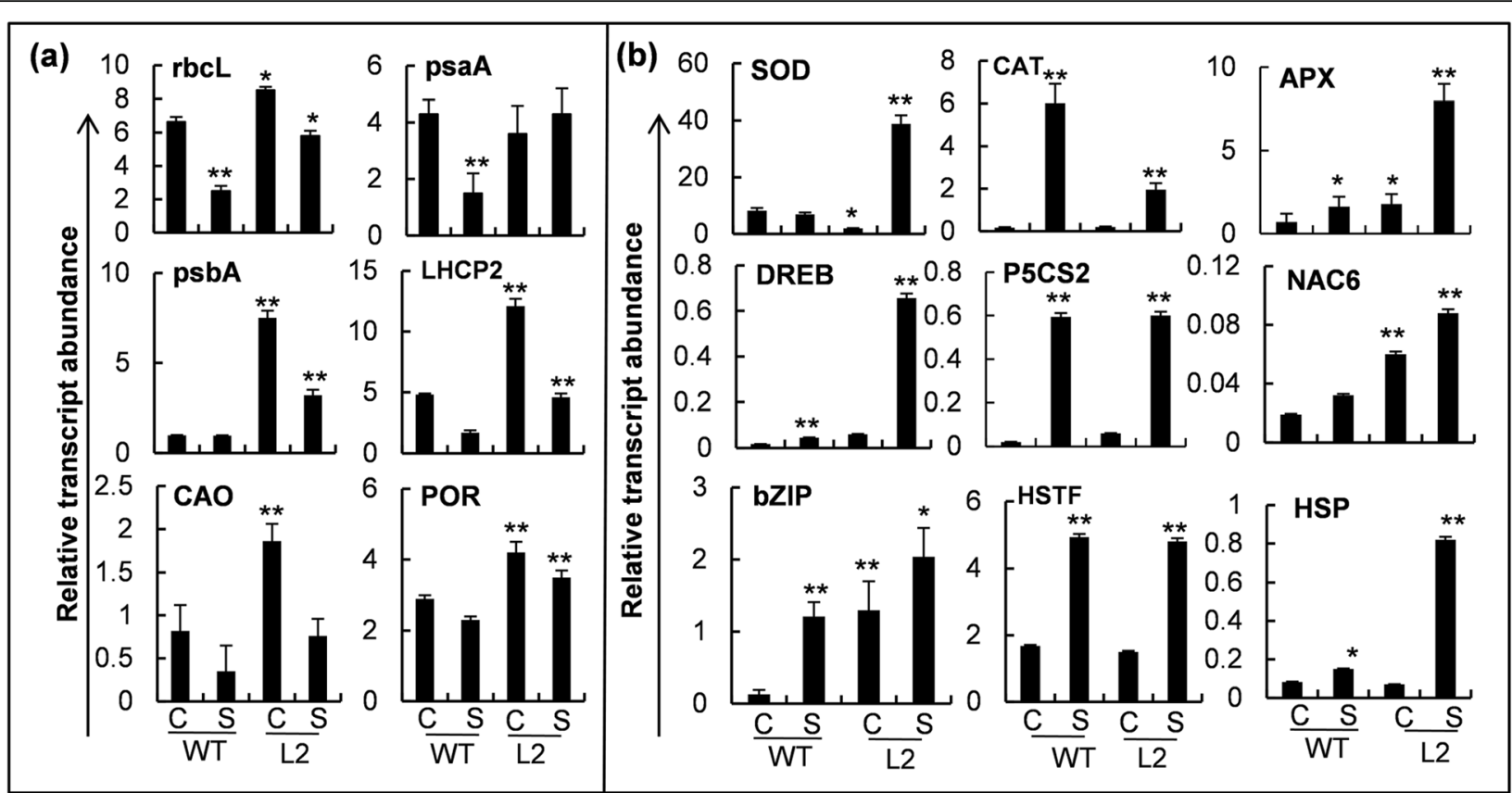

Fig. 6 Transcript abundance analysis for the various photosynthesis and stress-related genes in WT and OsHBP1b over-expressing plants under control and salinity. a Relative expression analysis of selected photosynthesis related genes such as ribulose bisphosphate carboxylase/oxygenase (RbcL), Photosystem I P700 chlorophyll a apoprotein A1 (PsaA), Photosystem II P680 chlorophyll a apoprotein A1 (PsbA), Light harvesting complex protein 2 (LHCP2), chlorophyllide a oxygenase (CAO) and P450 oxidoreductase (POR) in WT and OsHBP1b over-expressing plants. b Relative expression analysis of selected stress responsive gens (SOD, superoxide dismutase; APX, ascorbate peroxidise; CAT, catalase; bZIP, leucine zipper transcription factor; HSTF, heat shock transcription factor; HSP, heat shock protein; MT, metallothionine; DREB, dehydration responsive element binding protein; NAC TF, NAC domain containing transcription factors; P5CS, 2-pyrroline-5-carboxylate synthetase-2) in WT and OsHBP1b overexpressing plants. Expression analysis was done by quantitative real time PCR method. Expression of eEF gene was taken as reference and transcript abundance was calculated using $2^{-\Delta C T}$ method. Error bars show standard error from triplicate experiments. Single asterisk show significant difference at $p<0.005$ and double asterisk at $p<0.001$

both drought and high temperature stress (Additional file 2: Figure S2d and Additional file 3: Figure S3d). Significant increase in APX $(\sim 37 \%$ in drought and $35 \%$ in high temperature) and SOD (20\% in drought and $18 \%$ in high temperature) activity was evident in the $O s H B P 1 b$ over-expressing lines as compared to the WT, under drought and high temperature conditions (Additional file 2: Figure S2f, S2g, Additional file 3: Figure S3f and Additional file 3: Figure S3g). Under drought stress condition, the activity of catalase did not change significantly in WT and OsHBP1b over-expressing lines, whereas, high temperature could induce the CAT activity up to $31 \%$ (Additional file 2: Figure S2e and Additional file 3: Figure S3e). Since it is well established that bZIP proteins are strongly induced by ABA (Liu et al. 2014), the responsiveness of the OsHBP1b overexpressing and WT lines was also studied after treatment with 5 and $10 \mu \mathrm{M}$ of ABA. It was observed that, under $5 \mu \mathrm{m}$ of ABA, the WT plants possessed a lesser shoot and root length than the OsHBP1b overexpressing lines, whereas, OsHBP1b over-expressing lines could grow normally without any inhibition of shoot and root growth under similar conditions
(Additional file 4: Figure S4a and S4b). The fresh weight of the WT plant was also found to be less in comparison to the OsHBP $1 b$ over-expressing lines under $5 \mu \mathrm{M}$ of ABA treatment (Additional file 4: Figure S4c). It was also evident that $10 \mu \mathrm{M}$ of $\mathrm{ABA}$ affected the shoot and root growth severely, unlike the $O s H B P 1 b$ over-expressing lines which germinate and grow better even upon $10 \mu \mathrm{M}$ of ABA treatment (Additional file 4: Figure S4d and S4e). The fresh weight of the WT seedlings was also drastically decreased upon ABA treatment, as compared to the $O s H B P 1 b$ over-expressing lines (Additional file 4: Figure S4f). These results clearly suggest that the expression level of $O s H B P 1 b$ in rice is certainly linked with tolerance to multiple abiotic stresses which makes the plant tolerant to various stresses, unlike the WT plants.

\section{Discussion}

HBP1b protein is classified under bZIP transcription factors family due to its DNA binding specificity. OsHBP1b contains a highly conserved bZIP domain as evidence of a bZIP family protein (Busch and Sassone-Corsi 1990; Lakra et al. 2015). This protein has the highest affinity 
for 'ACGTCA' hexamer motif in the promoter regions of histone genes, and are reported to be participated in the regulation of histone gene transcription (Mikami et al. 1989; Tabata et al. 1991). We have cloned the full length $O s H B P 1 b$ gene in the plant transformation vector pCAMBIA 1304. Sequence analysis of OsHBP1b protein revealed that it possesses more than $94 \%$ similarity with the reported HBPs of barley and wheat (Lakra et al. 2015). OsHBP1b protein has been nuclear localized (Lakra et al. 2015) which was also in agreement with other reports (Mikami et al. 1989).

OsHBP1b is a Saltol QTL-localized gene found on chromosome number 1 in the rice genome. Previous reports have documented the constitutive and salinityinduced expression patterns for the various genes localized within the Saltol QTL (Soda et al. 2013; Nutan et al. 2017), which revealed $O s H B P 1 b$ gene to be induced under salinity in rice seedlings. This observation led us to hypothesize that $O s H B P 1 b$ might have a key function in rice salt stress tolerance. Subsequently, this hypothesis got strong support from the observation when the seedlings from the salt-tolerant genotype (Pokkali) showed elevated constitutive expression of OsHBP1b transcripts than the sensitive IR64 with further differential accumulation of transcripts under salinity stress (Lakra et al. 2015). Further analysis of the upstream and genic region of OsHBP1b confirmed significant differences in the single nucleotide polymorphisms (SNPs) and insertion-deletions (InDels) patterns in the contrasting genotypes IR64 and Pokkali (Additional file 6: Table S2). It is a fact that the bZIP TFs are indispensable in plant growth and development and their expression can be induced in response to abiotic and biotic stresses ( $\mathrm{E}$ et al. 2014). In the present study, we observed that $O s H B P 1 b$ is expressed during all the developmental stages of rice (Fig. 1a). However, this gene showed differential expression in various plant tissues when analysed using the publicly available microarray database and subsequent validation by quantitative realtime PCR (Fig. 1b and c). Several reports have shown that the expression of bZIP transcription factor genes gets altered in response to various abiotic stresses (Gao et al. 2011; Liu et al. 2014). Our results are in accordance with these reports where we found the induced expression of $O s H B P 1 b$ transcript in response to various abiotic stresses such as salinity, high temperature, drought or ABA (Fig. 1e-h). These results motivated us to investigate the function of rice $H B P 1 b$ towards enhancing abiotic stress tolerance in rice by over-expressing the gene using a strong promoter (CaMV35S).

In the present study, we over-expressed $O s H B P 1 b$ in a high yielding salt-sensitive rice IR64, with the purpose to dissect out the role of this gene towards multiple abiotic stress tolerance. It was found that OsHBP1b overexpressing single copy lines possess $4-5$ fold higher expression of $O s H B P 1 b$ transcript (Additional file 1: Figure S1) confirming the transgene functionality. It is well known that even though the response of plants towards abiotic stress is a multigenic trait, salt tolerant transgenic plants could be developed by introducing a single gene (SinglaPareek et al. 2001; Tripathi et al. 2016; Soda et al. 2018). Published literature has also shown that the plants ectopically-expressing bZIP transcription factors exhibit a positive effect on their growth, development and salinity tolerance (Gao et al. 2011; Liu et al. 2014; E et al. 2014; Lakra et al. 2015). Our findings are in agreement with these reports. We found better germination capacity, higher shoot growth, and higher fresh weight in OsHBP1b overexpressing lines than the WT, under control as well as salinity conditions (Fig. 3a, b, c, d and e). Larger parenchyma cells, longer roots, higher numbers of root branches were reported being responsible for providing dehydration stress tolerance by having more water holding capacity in rice plants (Ambavaram et al. 2014). In agreement to this study, we found better root morphology with longer root and more secondary branches in the transgenic plants under various abiotic stress conditions, in comparison with the WT plants (Fig. 3a, b and d, Additional file 2: Figure S2a and S2b, S3a and S3b and Additional file 4: Figure S4a and S4b). In addition, under control conditions, we also found the larger cortical region with larger parenchyma cells with accumulated callose (Fig. 5c) which might be providing limited access to ions and hence, providing salinity tolerance in the transgenic plants. Our finding of larger cortical parenchyma in transgenic plant is corroborated with a recent finding in wheat showing the genotype having larger root cortical cell required less energy to penetrate the hard soil and thus less translocation of carbohydrate to root is required which remains critical for plant survival under stress (Colombi et al. 2019). Larger metaxylem, which we have observed in transgenic rice root is reported to be a positive characteristic helping the plant under drought stress (Kadam et al. 2015). Various reports have stated the suberization, lignification and callose deposition in root cell under salinity stress to prevent the entry of harmful ions in the cell (Koyro 1997; Cui and Lee 2016; Hunter et al. 2019). Even though introduction of bZIP TF led to enhanced stress tolerance in plants, the mechanism of tolerance primarly remains unknown. Furthermore, characterization and role (if any) of OsHBP1b in stress response in rice has not been explored yet. This encouraged us to perform further biochemical and physiological analysis of the $O s H B P 1 b$ over-expressing rice, in response to salt stress as well as under other major abiotic stress conditions.

Treatment of WT and transgenic leaf segments in 100, 200 and $300 \mathrm{mM} \mathrm{NaCl}$, revealed early senescence of the WT leaf as compared to the OsHBP1b over-expressing 
plants (Fig. 2a). Further, the reduction in chlorophyll contents in the OsHBP1b transgenic lines was lesser (15-25\%) than the wild type counterparts (Fig. 2b). These results show a positive relationship between the expression of OsHBP1b and salt stress tolerance in leaf tissues. It has been established that the accumulation of ROS is connected to physiological balance and any fluctuations in ROS level can disturb the normal function of cellular machinery (Miller et al. 2010; Li et al. 2013). So, we checked the ROS level in plants by histochemical staining with NBT and DAB, which verified that under salt stress, the OsHBP1b over-expressing plants accrue less reactive oxygen molecules $\left(\mathrm{O}_{2}^{-}\right.$and $\left.\mathrm{H}_{2} \mathrm{O}_{2}\right)$ than wild type plants (Fig. 2c and d). It is also known that the level of ROS plays a crucial role in stress response in plant (Székely et al. 2008). For detoxification of ROS produced during stress, plants build up a multifaceted antioxidant arrangement, where several antioxidant proteins have a significant contribution towards protecting plant/cells against oxidative burst (Jaleel et al. 2009; Miller et al. 2010; Kumar et al. 2013). Under salinity, the activity of APX and SOD was found to be higher in the OsHBP1b over-expressing plants than the wild types (Fig. $3 j$ and k). Our work indicated that enhanced ROS scavenging through antioxidant enzymes might be an essential part of salinity protection machinery in the developed OsHBP1b transgenic lines. In agreement to our previous study (Lakra et al. 2015); insignificant change in CAT activity (Fig. 3i) under salt stress condition indicated the least participation of CAT in salinity tolerance system. Moreover, reports confirmed the induction of ROS scavenging genes after ectopic expression (Wu et al. 2008), representing the connection of transgene in the transcriptional regulation of antioxidant enzyme pool. In agreement with this, we have also observed induced expression of CAT, APX and SOD transcripts under salinity conditions (Fig. 6). Reports have claimed that under stress conditions, total protein level and percentage of electrolyte leakage changes drastically (Song et al. 2011; Gulen and Eris 2003). We observed a reduction in the percentage of electrolyte leakage in the OsHBP1b overexpressing transgenic plants (Fig. 3f), which is known to participate in plant stress tolerance mechanism in a constructive manner (Song et al. 2011). We observed a higher $\mathrm{K}^{+} / \mathrm{Na}^{+}$and higher protein content in the $O s H B P 1 b$ over-expressing lines when compared with the wild type plants (Fig. $3 g$ and h), which are also positive indicators of stress tolerance. Our result are in corroboration with other findings where enhanced tolerance in transgenic rice overexpressing a bZIP transcription factor (OsbZIP71) has been reported to confer salinity and drought tolerance in rice (Liu et al. 2014). Role of bZIP TF in stress tolerance has also been established by RNAi technology where silencing of similar bZIP1 domain containing gene PtabZIP1, AtbZIP1 and SibZIP1 from poplar, Arabidopsis and tomato results in enhanced sensitivity towards salinity and drought which further affects the overall plant growth and development (Sun et al. 2012; Dash et al. 2017; Zhu et al. 2018).

It has already been proven that salinity stress has a negative effect on the structure of the plant organelles (Pareek et al. 1997; Xu et al. 2008; Salama et al. 2012). Here, we found that over-expression of OsHBP1b could protect the cellular structure by maintaining the organellar membrane and thylakoid stacking, unlike the WT plants, where all the organelles including cell wall showed severe damage upon $200 \mathrm{mM} \mathrm{NaCl}$ treatment (Fig. 4a). Stomata of the OsHBP1b over-expressing lines were found to be protected which may contribute towards controlling the rate of transpiration under salinity conditions (Fig. 4b). These result are in harmony with the previous reports by Gao et al. (2011), where the closing of stomata was evident upon stress treatment.

Several studies have demonstrated that plants under abiotic stress conditions downregulate the expression of genes involved in photosynthesis and carbohydrate metabolism (Seki et al. 2002; Wong et al. 2006). Furthermore, transcription factors are also found to be involved in photosynthetic improvement when over-expressed (Saibo et al. 2009). In agreement with Saibo et al. (2009), we found increased chlorophyll fluorescence parameters, decreased non-photochemical quenching and increased photosynthesis activity in the OsHBP1b over-expressing lines as compared with the WT, under control as well as salinity conditions (Fig. 5). Furthermore, a recently published report showed that coordinated regulation of photosynthesis in rice could increase the environmental stress tolerance and yield related parameters (Ambavaram et al. 2014). Importantly, higher chlorophyll content and plant biomass were evident in the OsHBP1b overexpressing rice under salinity conditions (Fig. 2b), which are the major achievements of this study.

It is a well-established fact that TFs are present in all the organisms as they are the integral part of gene expression and regulation (Ashraf and Harris 2013; Joshi et al. 2016). Recently, the role of numerous TFs involved in regulation of the photosynthesis related-genes has been described (Saibo et al. 2009). Furthermore, it has been reported that bZIP transcription factor such as; LOG HYPOCOTYL 5 (HY5) is mainly involved in the regulation of $\mathrm{Chl} \mathrm{a} / \mathrm{b}$ binding gene expression, though it may also have an important contribution in abiotic stress tolerance (Saibo et al. 2009). Additionally, it has also been shown that this bZIP TF controls Rubisco small subunit $(\mathrm{rbcS1A})$ gene expression (Chattopadhyay et al. 1998; Lee et al. 2007). In agreement with these results, we also observed an increased expression of various 
photosynthesis related genes ( $\mathrm{rbcL}$, psbA, LHCP2, CAO and POR) in the OsHBP1b over-expressing plants in comparison with WT (Fig. 6a). Furthermore, it has also been revealed that the expression of these photosynthetic genes decreased under salinity stress in WT plants where as OsHBP1b over-expressing lines maintained the gene expression to the level of WT control plants, even under salinity (Fig. 6a).

Apart from maintained photosynthesis rate, abiotic stress tolerance is an essential character to get higher plant yield under unfavourable environmental conditions. Modulation in the expression of stress-related genes is another way to develop stress tolerance in the plants. As it is known that abiotic stress tolerance is a multigenic trait, non-regulatory genes related to stress tolerance do not always meet the aim to get abiotic stress tolerant plants after over-expression. To overcome this, TFs that regulate several genes have often used to increase stress tolerance in a broader response (Nelson et al. 2009). Many transcription factors such as; bZIP (Zhang et al. 2008), NAC (Hu et al. 2006), MYB (Vannini et al. 2004) and zinc finger (Kim et al. 2001), have been found to be involved in providing multiple stress tolerance by regulating various important functions and manipulating many stress-responsive genes. Moreover, some researchers have established that bZIP TFs help to regulate stress-related genes under control as well as abiotic stress situations (Huang et al. 2010; Yáñez et al. 2009; ZG et al. 2014). Interestingly, we also found altered expression of various stressrelated transcripts including antioxidant genes; heat stress related genes and drought stress related genes, in $O s H B P 1 b$ over-expressing lines under stress conditions (Fig. 6). These results suggested that over-expression of $O s H B P 1 b$ was able to alter the expression of the stress-responsive genes and are positively involved in providing higher photosynthesis and stress tolerance under salinity.

As abiotic stresses are interrelated to each other, we further decided to verify the drought, high temperature and ABA responsiveness of the OsHBP1b overexpressing lines with a comparison to its WT counterpart. Our results clearly suggested that the OsHBP $1 b$ over-expressing lines have a better phenotype, antioxidant activity and photosynthesis rate under drought and high-temperature stress conditions (Additional file 2: Figure S2 and Additional file 3: Figure S3). Moreover, bZIP transcription factors have been recognized as important players in ABA signalling pathway of abiotic stress (Liu et al. 2014). In accordance, we have found better shoot and root growth of OsHBP1b overexpressing lines even with $10 \mu \mathrm{M}$ of $\mathrm{ABA}$ treatment, while at this particular concentration of ABA, WT plants could not even germinate (Additional file 4: Figure S4). This proves a crosstalk between the stress response and harmones in plants (Karan et al. 2009; Nongpiur et al. 2012).

\section{Conclusions}

The function of bZIP subfamily in rice is less explored. Here, we have investigated the possible role of bZIP subfamily protein (OsHBP1b) in rice and, taken together, our results describe the importance of $O s H B P 1 b$ in relation to stress tolerance and plant yield. Over-expression of $O s H B P 1 b$ in rice, under the control of CaMV35S promoter, signifies its own role in stress management by minimizing the level of ROS, increasing antioxidant enzyme activity, maintaining organelle structure, increasing photosynthesis and modulating the level of photosynthesis and stress-related transcripts. Furthermore, ABA receptiveness of the generated $O s H B P 1 b$ transgenic rice plants suggests that the stress tolerance behaviour in these lines is related to the ABA signal transduction pathway. Nevertheless, the reality that $O s H B P 1 b$ over-expression noticeably showed minimum impact on grain filling and panicle length of rice under salinity suggests that this gene could be used in other plants species to increase their productivity under salinity and other unfavourable environmental situations.

\section{Methods \\ Plant material and growth conditions}

Rice (Oryza sativa L., cv. IR64) was used for the expression analysis of OsHBP1b under control and various abiotic stress conditions. Rice seeds were surface sterilized and grown by following the protocol as mentioned in Lakra et al. (2015). 7 days old plants were exposed to salinity, drought, high temperature and ABA stress and use for further analysis using standard protocols, mentioned in following section. For further study of various growth parameters and seed harvesting, 25 days old $\mathrm{T}_{1}$ or $\mathrm{T}_{2}$ OsHBP1b overexpressing rice lines were transferred to green house.

\section{Stress treatments}

In order to determine the transcript abundance, $7 \mathrm{~d}$ old rice seedlings grown hydroponically under control conditions were transferred to a solution of $200 \mathrm{mM} \mathrm{NaCl}$ up to $24 \mathrm{~h}$. Samples were collected from $0 \mathrm{~h}, 0.5 \mathrm{~h}, 12 \mathrm{~h}$, and $24 \mathrm{~h}$ after stress treatment. For high temperature stress, rice seedlings were transferred to a growth chamber maintained at $45^{\circ} \mathrm{C}$ and samples were harvested after $0 \mathrm{~h}, 0.5 \mathrm{~h}, 3 \mathrm{~h}$ and $6 \mathrm{~h}$. Similarly, for drought stress, the seedlings grown on $5 \%$ PEG were used and for ABA seedlings were treated with $10 \mu \mathrm{M}$ ABA and samples were harvested after $0 \mathrm{~h}, 0.5 \mathrm{~h}, 12$ $\mathrm{h}$ and $24 \mathrm{~h}$ of stress imposition. A set of seedlings were alwayes kept under control conditions for the same duration. For various morphological and physiological parameters (shoot length, root length, fresh weight, electrolyte leakage, $\mathrm{K}^{+} / \mathrm{Na}^{+}$ratio, total protein and antioxidant enzymes activity) of $O s H B P 1 b$ over-expressing rice in response to salinity, $7 \mathrm{~d}$ old $\mathrm{T}_{2}$ rice seedlings grown hydroponically under control conditions were transferred to solution of $200 \mathrm{mM} \mathrm{NaCl}$ up to $3 \mathrm{~d}$. Samples were collected after $72 \mathrm{~h}$ of stress treatment. 
Tissue-specific OsHBP1b expression and expression of $O s H B P 1 b$ after transgene insertion were done by using $24 \mathrm{~h}$ $\mathrm{NaCl}$ treated plants samples. The expression of stress-related and photosynthesis-related genes was studied using $72 \mathrm{~h}$ stress-treated samples. For assessment of other parameters like organellar organization, stomatal structure, $\mathrm{H}_{2} \mathrm{O}_{2}$, superoxide level, and photosynthetic parameters and yield related parameters, $90 \mathrm{~d}$ old plants were treated with $200 \mathrm{mM} \mathrm{NaCl}$ solution for 15 days, followed by recovery with water. For the leaf segment assay, leaf segments $(1 \mathrm{~cm}$ length) were incubated (for $72 \mathrm{~h}$ ) with liquid Yoshida medium supplemented with $100 \mathrm{mM}$ or $200 \mathrm{mM}$ or $300 \mathrm{mM} \mathrm{NaCl}$.

For the assessment of growth and physiological parameters of $O s H B P 1 b$ over-expressing rice plants under multiple abiotic stresses, $\mathrm{T}_{2}$ rice seedlings were grown under control conditions inside a growth chamber for 7 $\mathrm{d}$ and subsequently, treated with either ABA (5 or $10 \mu \mathrm{M}$ for $72 \mathrm{~h}$ ) or PEG (5\%, for drought stress for $72 \mathrm{~h}$ ) or high temperature $\left(45^{\circ} \mathrm{C}\right.$ for $12 \mathrm{~h}$, subsequent recovery for $3 \mathrm{~d}$ ) in a hydroponic setup.

\section{Transcript abundance analysis}

The transcript abundance for OsHBP1b at different stages of development was analyzed using the publicly available database (https://www.genevestigator.com/gv/) using default parameters. The expression analysis of various genes was carried out by quantitative real-time PCR method as described earlier (Lakra et al. 2015; Nutan et al. 2017). Primer pairs used for transcript expression were given in Additional file 5: Table S1.

\section{Raising of transgenic rice plants over-expressing OsHBP1b and confirmation of transgenic lines}

Cloning and transformation of OsHBP1b gene was done by following the earlier protocols where the expression of the gene is controlled by CaMV $35 \mathrm{~S}$ constitutive promoter (Lakra et al. 2015). Callus induction, callus transformation, and plant regeneration from transformed callus were done by following the protocol of Sahoo et al. (2011). OsHBP1b over-expressing rice plants were selected on MS media having $50 \mathrm{mg} / \mathrm{l}$ hygromycin. The $O s H B P 1 b$ over-expressing rice plants which grew properly in hygromycin-supplemented media were screened through tissue PCR with a combination of gene-specific forward and vector specific reverse primer pairs. Genomic DNA from wild type IR64 and pCAMBIA1304OsHBP1b plasmid were taken as negative and positive control respectively. 8 plants positive for the presence of the transgene are shown here as they amplified the desired band from the genomic DNA of the putative OsHBP1b over-expressing lines. Segregation analysis of $\mathrm{T} 1$ transgenic seeds were done in presence of hygromycin showing their single copy nature (Additional file 7: Table S3). Transgenic nature of OsHBP1b over- expressing plants was also confirmed by Southern hybridization method as described by Sambrook and Russell (2001), where EcoRI was used to digest the genomic DNA and OsHBP1b was used as probe.

\section{Western blot analysis}

The relative concentration of HBP1b proteins in the leaf extracts of WT and transgenic plants was analyzed by western blot analysis. The crude protein extracted in 50 $\mathrm{mM} \mathrm{K}_{2} \mathrm{PO} 4$ buffer (pH 7.0) containing $1 \mathrm{mM}$ PMSF was separated on a $10 \%$ SDS-PAGE and was transferred onto the activated immunoblot PVDF membranes (Sigma) with semi-dry blotting apparatus according to the provided instructions. To check the abundance of HBP1b protein, the blots were treated with protein-specific polyclonal antibodies (Lakra et al. 2015) separately and developed with a SuperECL Plus kit (Thermo Scientific, Pierce, USA), and the signal was exposed with X-ray film.

\section{Physiological and biochemical analysis for the transgenic plants \\ $\mathrm{Na}^{+}$and $\mathrm{K}^{+}$measurement}

To determine the leaf $\mathrm{Na}^{+}$and $\mathrm{K}^{+}$content, $100 \mathrm{mg}$ of tissues from $\mathrm{NaCl}$-treated and untreated plants were digested with $\mathrm{HNO}_{3}(0.1 \%)$. Determination of $\mathrm{K}^{+}$and $\mathrm{Na}^{+}$content was done by atomic absorption spectrometer (Central Instrument Facility, Jawaharlal Nehru University) following the protocol of Lakra et al. (2015).

\section{Electrolyte leakage}

Analysis of electrolyte leakage was carried out following the protocol described in Lakra et al. (2015). $100 \mathrm{mg}$ leaf samples were taken for this analysis and the formula used for measurement of relative electrical conductivity is: electrolyte leakage $(\%)=\mathrm{E} 1 / \mathrm{E} 2 * 100$ where $\mathrm{E} 1$ is electrical conductivity and E2 is total conductivity.

\section{Chlorophyll estimation}

Chlorophyll content in control and $\mathrm{NaCl}$ treated tissues was determined following the method of Arnon (1949) using UV/Vis spectrophotometer.

\section{Determination of $\mathrm{H}_{2} \mathrm{O}_{2}$ and $\mathrm{ROS}$ levels}

Level of reactive oxygen species (ROS) in the leaves was estimated through histochemical staining assay using NBT (nitro blue tetrazolium) as described by Lakra et al. (2015). In situ detection of hydrogen peroxide $\left(\mathrm{H}_{2} \mathrm{O}_{2}\right)$ was performed by staining the tissue with DAB (SigmaAldrich) as suggested (Bindschedler et al. 2006). Steadystate levels of $\mathrm{H}_{2} \mathrm{O}_{2}$ in leaves were determined following FOX-1 method (Sahu et al. 2010). 


\section{Total protein measurement and activity assay of antioxidant enzyme}

Total proteins extraction and antioxidant enzyme activity assays were performed following the previously published method (Lakra et al. 2015).

\section{Electron microscopy}

Young leaf sections $(0.5-1 \mathrm{~cm})$ were fixed first in a solution of $2 \%$ glutaraldehyde and then in $1 \% \mathrm{OsO}_{4}$. Vacuum was applied to the dipped samples to ensure the penetration of fixative solution to the leaf pieces. Tissues were stained with uranyl acetate following dehydration in ethanol and embedded in Spurr's medium. Samples were then sectioned and stained once more and observed under an electron microscope (JEOL) in Advanced Instrument Research Facility, Jawaharlal Nehru University. For ultrastructure analysis of leaf surface, the samples were prepared following the protocol of Zhou et al. (2013) and examined in an XL-30-ESEM (ZEISS) in Advanced Instrument Research Facility, Jawaharlal Nehru University.

\section{Root anatomy study}

The root of WT and OsHBP1b over-expressing rice seedlings were cut by free hand to get the transverse sections. The sections were stained with safranin $(0.25 \%$ $\mathrm{w} / \mathrm{v})$ and methylene blue $(0.25 \% \mathrm{w} / \mathrm{v})$ for tissue demarcation and mounted in glycerine $(10 \% \mathrm{v} / \mathrm{v})$ and observed under light microscope (Leica) and photographs were taken with a digital camera fitted to the microscope.

\section{Chlorophyll a fluorescence and photosynthesis related study} Chlorophyll $a$ fluorescence and photosynthesis of the leaf of mature plants (third leaf from the top from 100 days old plants) was monitored at $25^{\circ} \mathrm{C}$ undersupplied $\mathrm{CO}_{2}$ concentration $(400 \mathrm{ppm})$, by using Infra-Red Gas Analyser (IRGA, LICOR-6400XT). Leaves were darkadapted for $30 \mathrm{~min}$ prior to measurement of fluorescence. The minimal level of dark fluorescence $\left(\mathrm{F}_{0}\right)$ was measured under weak modulated light and the maximal intensity of fluorescence $\left(\mathrm{F}_{\mathrm{m}}\right)$ was evoked by application of a short saturating light pulse $\left(10,000 \mu \mathrm{mol} \mathrm{m}^{-2} \mathrm{~s}^{-1}\right)$. The maximal steady state photochemical efficiency (i.e. intrinsic quantum yield under dark adapted condition) was indicated by $\left(F_{v} / F_{m}\right)$, where $F_{v}=F_{m}-F_{0}$. The electron transport rate (ETR) was estimated from the equation ETR = quantum yield * photosynthetic photon flux density (PPFD) * $0.84 * 0.5$, where 0.84 represents the standard leaf absorptance and 0.5 denotes that the rate of PS-I photochemistry is in a match with PS-II. Photosynthesis of WT and OsHBP1b over-expressing plants was measured on a sunny day without dark adaptation.

\section{SNPs and InDels analysis}

The analysis of SNPs and InDels in IR64 and Pokkali was performed using the Rice SNP discovery database (http://14.139.61.3:8080/mjain/ricesnp/index.html) which holds the re-sequenced data for the rice cultivars (Jain et al. 2014).

\section{Statistical analysis}

Readings were obtained from a minimum of three biological replicates for each experiment. Data were analysed using ANOVA by GraphPad InStat3 software.

\section{Additional files}

\begin{abstract}
Additional file 1: Figure S1. Confirmation and molecular analysis of transgenic rice lines over-expressing OsHBP1b. (a) Tissue PCR analysis showed eight positive rice lines over-expressing OsHBP1b. Vector specific forward and gene specific reverse primer pair (F1R1) has been used here. (b) Southern blot analysis showed pattern of digestion (left) and copy number (right) of transgene in the OsHBP1b over-expressing plants. Two lines (L2 and L7) showed intense and clear band having a single copy of transgene and hence, further experimental analysis were done by using $\mathrm{L} 2$ and L7 lines. (c) Fold change in expression of OsHBP1b in OsHBP1b over-expressing plant as compared with WT, analyzed through quantitative real-time PCR. Expression of eEF was taken as reference and fold change was calculated using $2^{-\Delta \Delta C T}$ method. (d) Western blot analysis showed higher OsHBP1b protein accumulation in the OsHBP1b over-expressing plants as compared with the WT. Error bars show standard error from triplicate experiments. Single asterisk show significant difference at $p<0.005$ and double asterisk at $p<0.001$. (TIF $7325 \mathrm{~kb}$ )
\end{abstract}

Additional file 2: Figure S2. Assessment of drought stress tolerance of OsHBP1b over-expressing rice seedlings through morphological, physiological and enzymatic analysis. (a) Photograph of WT and OsHBP1b over-expressing plants under drought conditions. Note the prominent difference in root morphology where OsHBP16 over-expressing plants have longer roots with more secondary branches as compared to the WT under drought stress. Bar diagrams showing (b) shoot and root length, (c) fresh weight, (d) Fv/Fm, (e) CAT activity, (f) APX activity and (g) SOD activity measured in the leaves of seedlings after 3 days of drought stress. The data represent means \pm SE of three biological replicates. Single asterisk show significant difference at $p<0.005$ and double asterisk at $p<0.001$. (TIF $8735 \mathrm{~kb}$ )

Additional file 3: Figure S3. Assessment of high-temperature $\left(45^{\circ} \mathrm{C}\right)$ stress tolerance of OsHBP1b over-expressing rice seedlings through morphological, physiological and enzymatic analysis. (a) Photograph of WT and OsHBP16 over-expressing plants after $12 \mathrm{~h}$ of high-temperature $\left(45^{\circ} \mathrm{C}\right)$ conditions and subsequent recovery for 3 days. Note the prominent difference in root morphology where OsHBP1b over-expressing plants have longer roots with more secondary branches as compared to the WT under high temperature stress. Bar diagrams showing (b) shoot and root length, (c) fresh weight, (d) Fv/Fm, (e) CAT activity, (f) APX activity and (g) SOD activity measured in leaves of seedlings after 3 days of recovery. The data represent means \pm SE of three biological replicates. Single asterisk show significant difference at $p<0.005$ and double asterisk at $p<0.001$. (TIF $8178 \mathrm{~kb}$ )

Additional file 4: Figure S4. Assessment of ABA responsiveness of OsHBP1b over-expressing rice seedlings through morphological and physiological analysis. (a) Photograph of WT and OsHBP16 over-expressing plants in response to exogenous ABA ( $5 \mathrm{uM})$. Note the prominent difference in root morphology where OSHBP1b over-expressing plants have longer roots with more secondary branches as compared to the WT in response to ABA. Bar diagrams showing (b) shoot and root length, (c) fresh weight, (d) Photograph of WT and OsHBP1b over-expressing plants in response to exogenous ABA (10 uM). Bar diagrams showing (e) shoot and root length, (f) fresh weight. The data represent means \pm SE of three biological replicates. 
Single asterisk show significant difference at $p<0.005$ and double asterisk at $p<0.001$. (TIF $9550 \mathrm{~kb}$ )

Additional file 5: Table S1. List of primers used in this study and their sequences ( $5^{\prime}$ to $3^{\prime}$ ). (DOCX $16 \mathrm{~kb}$ )

Additional file 6: Table S2. SNPs and InDels analysis of Saltol QTL localized gene OsHBP1b (LOC_Os01g17260) in IR64 and Pokkali genotypes of Oryza sativa L. (DOCX $21 \mathrm{~kb}$ )

Additional file 7: Table S3. Segregation analysis of transgenic lines over-expressing OsHBP1b. (DOCX $15 \mathrm{~kb}$ )

\section{Acknowledgements}

Research support by UGC as Dr. D.S. Kothari Post-Doctoral Fellowship to PD is highly acknowledged.

\section{Authors' contributions}

PD performed experiments, analyzed data and wrote the manuscript. NL performed initial experiments. KKN performed SNPS and InDels analysis and helped in revision of the manuscript. AP conceived and designed the study. AP and SLS-P finalized the manuscript. All authors read and approved the final manuscript.

\section{Funding}

AP is thankful to the Department of Science and Technology, Government of India, for the funding received for the lab through Indo-Sri Lanka joint project and DST-PURSE.

\section{Availability of data and materials}

The data sets supporting this article are included in the article and in the additional files.

\section{Ethics approval and consent to participate}

Not applicable.

\section{Consent for publication}

Not applicable.

\section{Competing interests}

The authors declare that they have no competing interests.

\section{Author details}

${ }^{1}$ Stress Physiology and Molecular Biology Laboratory, School of Life Sciences, Jawaharlal Nehru University, New Delhi 110067, India. 2Plant Stress Biology, International Centre for Genetic Engineering and Biotechnology, Aruna Asaf Ali Road, New Delhi 110067, India.

Received: 19 January 2019 Accepted: 11 July 2019

Published online: 02 August 2019

\section{References}

Alves MS, Dadalto SP, Goncalves AB, De Souza GB, Barros VA, Fietto LG (2013) Plant bZIP transcription factors responsive to pathogens. Int J Mol Sci 14: $7815-7828$

Ambavaram MMR, Basu S, Krishnan A, Ramegowda V, Batlang U, Rahman L, Niranjan B, Pereira A (2014) Coordinated regulation of photosynthesis in rice increases yield and tolerance to environmental stress. Nat Commun 5:5302

Arnon DI (1949) Copper enzymes in isolated chloroplasts Polyphenoloxidase in Beta vulgaris. Plant Physiol 24:1-15

Ashraf M, Harris PJC (2013) Photosynthesis under stressful environments: an overview. Photosynthetica 51:163-190

Bindschedler LV, Dewdney J, Blee KA, Stone JM, Asai T, Plotnikov J, Denoux C, Hayes T, Gerrish C, Davies DR, Ausubel FM, Bolwell GP (2006) Peroxidasedependent apoplastic oxidative burst in Arabidopsis required for pathogen resistance. Plant J 47:851-863

Bray E, Shih TY, Moses M, Cohen A, Imai R, Plant A (1999) Water-deficit induction of atomato $\mathrm{H} 1$ histone requires abscisic acid. Plant Growth Regul 29:35-46

Busch SJ, Sassone-Corsi P (1990) Dimers leucine zippers and DNA-binding domains. Trends Genet 6:36-40
Chattopadhyay S, Ang LH, Puente P, Deng XW, Wei N (1998) Arabidopsis bZIP protein HY5 directly interacts with light-responsive promoters in mediating light control of gene expression. Plant Cell 10:673-683

Colombi T, Herrmann AM, Vallenback P, Keller T $(2019,2019)$ Cortical cell diameter is key to energy costs of root growth in wheat. Plant Physiology. https://doi.org/10.1104/pp.19.00262

Cramer GR, Urano K, Delrot S, Pezzotti, Shinozaki K (2011) Effects of abiotic stress on plants: a systems biology perspective. BMC Plant Biol 11:163

Cui W, Lee JY (2016) Arabidopsis callose synthases CalS1/8 regulate plasmodesmal permeability during stress. Nature Plants 2(5):16034

Dash M, Yordanov YS, Georgieva T, Tschaplinski TJ, Yordanova E, Busov V (2017) Poplar PtabZIP1-like enhances lateral root formation and biomass growth under drought stress. Plant J 89(4):692-705

Gao SQ, Chen M, Xu ZS, Zhao CP, Li L, Xu HJ, Tang YM, Zhao X, Ma YZ (2011) The soybean GmbZIP1 transcription factor enhances multiple abiotic stress tolerances in transgenic plants. Plant Mol biol 75:537-553. https://doi.org/10.1007/s11103-011-9738-4

Gulen H, Eris A (2003) Some physiological changes in strawberry (Fragaria x ananassa cvCamarosa) plants under heat stress. J Hortic Sci Biotechnol 78: 894-898

Hu H, Dai M, Yao J, Xiao B, Li X, Zhang Q et al (2006) Overexpressing a NAM ATAF and CUC (NAC) transcription factor enhances drought resistance and salt tolerance in rice. Proc Natl Acad Sci USA 103:1 2987-12992. https://doi.org/10.1073/pnas060488210

Huang XS, Liu JH, Chen XJ (2010) Overexpression of PtrABF gene a bZIP transcription factor isolated from Poncirus trifoliata enhances dehydration and drought tolerance in tobacco via scavenging ROS and modulating expression of stress-responsive genes. BMC Plant Biol 10:230

Hunter K, Kimura S, Rokka A, Tran HC, Toyota M, Kukkonen JP, Wrzaczek M (2019, 2019) CRK2 enhances salt tolerance by regulating callose deposition in connection with PLDa1. Plant Physiology. https://doi.org/1 0.1104/pp.19.00560

Jain M, Moharana KC, Shankar R, Kumari R, Garg R (2014) Genome wide discovery of DNA polymorphisms in rice cultivars with contrasting drought and salinity stress response and their functional relevance. Plant Biotechnol J 12:253-264

Jakoby M, Weisshaar B, Droge-Laser W, Vicente-Carbajosa J, Tiedemann J, Kroj T, Parcy F (2002) bZIP transcription factors in Arabidopsis. Trends Plant Sci 7:106-111

Jaleel CA, Manivannan P, Wahid A, Farooq M, Al-juburi JH, Somasundaram R et al (2009) Drought stress in plants: a review on morphological characteristics and pigments composition. Inter J Agric Biol 11:100-105

Kadam NN, Yin X, Bindraban PS, Struik PC, Jagadish KS (2015) Does morphological and anatomical plasticity during the vegetative stage make wheat more tolerant of water deficit stress than rice? Plant Physiol 167(4):1389-1401

Kim JC, Lee SH, Cheong YH, Yoo CM, Lee SI et al (2001) A novel cold-inducible zinc finger protein from soybean SCOF-1 enhances cold tolerance in transgenic plants. Plant J 25:247-259

Koyro HW (1997) Ultrastructural and physiological changes in root cells of Sorghum plants (Sorghum bicolorxS. sudanensis cv. Sweet Sioux) induced by $\mathrm{NaCl}$. J Exp Bot 48:693-706

Kumar RR, Goswami S, Singh K, Rai GK, Rai RD (2013) Modulation of redox signal transduction in plant system through induction of free radical /ROS scavenging redox-sensitive enzymes and metabolites. Aus J Crop Sci 7:1744-1751

Kumari S, Sabharwal VP, Khushwa HR, Sopory SK, Singla-Pareek SL, Pareek A (2009) Transcriptome map for seedling stage specific salinity stress response indicates a specific set of genes as candidate for saline tolerance in Oryza sativa L. Funct Integr Genomics 9:109-123

Lakra N, Nutan KK, Das P, Anwar K, Singla-Pareek SL, Pareek A (2015) A nuclearlocalized histone-gene binding protein from rice (OsHBP1b) functions in salinity and drought stress tolerance by maintaining chlorophyll content and improving the antioxidant machinery. J Plant Physiol 176:36-46

Lee J, He K, Stolc V, Lee H, Figueroa P, Gao Y, Tongprasit W, Zhao H, Lee I, Deng XW (2007) Analysis of transcription factor HY5 genomic binding sites revealed its hierarchical role in light regulation of development. Plant Cell 19:731-749

Lee TI, Johnstone SE, Young RA (2006) Chromatin immunoprecipitation and microarray-based analysis of protein location. Nat Protoc 1:729-748

Li XY, Liu X, Yao Y, Li YH, Liu S, He CY, Li JM, Lin YY, Li L (2013) Overexpression of Arachis hypogaea AREB1 gene enhances drought 
tolerance by modulating ROS scavenging and maintaining endogenous ABA content. Int J Mol Sci 14:12827-12842

Liao Y, Zou HF, Wei W, Hao YJ, Tian AG, Huang J, Liu YF, Zhang JS, Chen SY (2008) Soybean GmbZIP44 GmbZIP62 and GmbZIP78 genes function as negative regulator of $A B A$ signaling and confer salt and freezing tolerance in transgenic Arabidopsis. Planta 228:225-240

Liu C, Mao B, Ou S, Wang W, Liu L, Wu Y, Chu C, Wang X (2014) OsbZIP71 a bZIP transcription factor confers salinity and drought tolerance in rice. Plant Mol Biol 84:19-36

Mikami K, Sakamoto A, Takase H, Tabata T, Iwabuchi M (1989) Wheat nuclear protein HBP-1 binds to the hexameric sequence in the promoter of various plant genes. Nucleic Acids Res 17:9707-9717

Miller G, Suzuki N, Ciftci-Yilmaz S, Mittler R (2010) Reactive oxygen species homeostasis and signalling during drought and salinity stresses. Plant Cell Environ 33:453-467

Nelson SM, Park L, Stenkamp DL (2009) Retinal homeobox 1 is required for retinal neurogenesis and photoreceptor differentiation in embryonic zebrafish. Dev biol 328:24-39. https://doi.org/10.1016/jydbio200812040

Nijhawan A, Jain M, Tyagi AK, Khurana JP (2008) Genomic survey and gene expression analysis of the basic leucine zipper transcription factor family in rice. Plant Physiol 146:333-350

Nutan KK, Kushwaha HR, Singla-Pareek SL, Pareek A (2017) Transcription dynamics of Saltol QTL localized genes encoding transcription factors reveals their differential regulation in contrasting genotypes of rice. Funct Integr genomics 17:69-83

Pareek A, Sopory SK, Bohnert HJ, Govindjee (2010) Abiotic stress adaptation in plants: physiological. Molecular and Genomic Foundation Springer, Berlin

Ramachandran S, Hiratsuka K, Chua NH (1994) Transcription factors in plant growth and development. Curr Opin Genet Dev 4(5):642-646

Sahoo KK, Tripathi AK, Pareek A, Sopory SK, Singla-Pareek SL (2011) An improved protocol for efficient transformation and regeneration of diverse indica rice cultivars. Plant Methods 7:49-58

Sahu S, Das P, Ray M, Sabat SC (2010) Osmolyte modulated enhanced rice leaf catalase activity under salt-stress. Adv Biosci Biotechnol 1:39-46

Saibo NJ, Lourenço T, Oliveira MM (2009) Transcription factors and regulation of photosynthetic and related metabolism under environmental stresses. Ann Bot 103:609-623

Salama YAM, Hassan NMK, Saleh SA, Zaki MF (2012) Zinc amelioration effects on tomato growth and production under saline water irrigation conditions. J Appl Sci Res 8(12):5877-5885

Sambrook J, Russell DW (2001) Rapid isolation of yeast DNA molecular cloning a laboratory manual 3rd ed. Cold Spring Harbor laboratory press, New York

Schultz TF, Spiker S, Quatrano RS (1996) Histone H1 enhances the DNA binding activity ofthe transcription factor EmBP-1. J Biol Chem 271:25742-25745

Seki M, Narusaka M, Ishida J, Nanjo T, Fujita M, Oono Y, Kamiya A, Nakajima M, Enju A, Sakurai T, Satou M, Akiyama K, Taji T, Yamaguchi-Shinozaki K, Carninci P, Kawai J, Hayashizaki Y, Shinozaki K (2002) Monitoring the expression profiles of 7000 Arabidopsis genes under drought, cold and high-salinity stresses using a full-length cDNA microarray. Plant J 31:279-292

Singla-Pareek SL, Reddy MK, Sopory SK (2001) Transgenic approach towards developing abiotic stress tolerance in plants. Proc Ind Nat Sci Acad 67: 265-284

Soda N, Gupta BK, Anwar K, Sharan A, Govindjee, Singla-Pareek SL, Pareek A (2018) Rice intermediate filament, OsIF, stabilizes photosynthetic machinery and yield under salinity and heat stress. Sci Rep 8(1):4072

Soda N, Kushwaha HR, Soni P, Singla-Pareek SL, Pareek A (2013) A suite of new genes defining salinity stress tolerance in seedlings of contrasting rice genotypes. Funct Integr Genomics 13(3):351-365

Song Q, Palikkuth S, Bossuyt J, Bers DM, Robia SL (2011) Phosphomimetic mutations enhance oligomerization of phospholemman and modulate its interaction with the Na/K-ATPase. J Biol Chem 286:9120-9126

Sun X, Li Y, Cai H, Bai X, Ji W, Ding X, Zhu Y (2012) The Arabidopsis AtbZIP1 transcription factor is a positive regulator of plant tolerance to salt, osmotic and drought stresses. J Plant Res 125(3):429-438

Székely G, Abrahám E, Cséplo A, Rigó G, Zsigmond L, Csiszár J, Ayaydin F, Strizhov N, Jásik J, Schmelzer E, Koncz C, Szabados L (2008) Duplicated P5CS genes of Arabidopsis play distinct roles in stress regulation and developmental control of proline biosynthesis. Plant J 53:11-28

Tabata T, Nakayama T, Mikami K, Iwabuchi M (1991) HBP-1a and HBP-1b: leucine zipper-type transcription factors of wheat. EMBO J 10:1459-1467
Tripathi AK, Pareek A, Singla-Pareek SL (2016) A NAP-family histone chaperone functions in abiotic stress response and adaptation. Plant Physiol 171(4): 2854-2868

Vannini C, Locatelli F, Bracale M, Magnani E, Marsoni M, Osnato M, Mattana M, Baldoni E, Coraggio I (2004) Overexpression of the rice Osmyb4 gene increases chilling and freezing tolerance of Arabidopsis thaliana plants. Plant J 37:115-127

Verslues PE, Agarwal M, Katiyar-Agarwal S, Zhu J, Zhu JK (2006) Methods and concepts in quantifying resistance to drought salt and freezing abiotic stresses that affect plant water status. Plant J 45:523-539

Wong CE, Li Y, Labbe A, Guevara D, Nuin P, Whitty B, Diaz C, Golding GB, Gray GR, Weretilnyk EA, Griffith M, Moffatt BA (2006) Transcriptional profiling implicates novel interactions between abiotic stress and hormonal responses in Thellungiella, a close relative of Arabidopsis. Plant Physiol 140:1437-1450

Wu L, Zhang Z, Zhang H, Wang XC, Huang R (2008) Transcriptional modulation of ethylene response factor protein JERF3 in the oxidative stress response enhances tolerance of tobacco seedlings to salt, drought, and freezing. Plant Physiol 148:1953-1963

Xu H, Jiang X, Zhan K, Cheng X, Chen X, Pardo JM, Cui D (2008) Functional characterization of a wheat plasma membrane $\mathrm{Na}^{+} / \mathrm{H}^{+}$antiporter in yeast. Arch Biochem Biophys 473:8-15

Yáñez M, Cáceres S, Orellana S, Bastías A, Verdugo I, Ruiz-Lara S, Casaretto JA (2009) An abiotic stress-responsive bZIP transcription factor from wild and cultivated tomatoes regulates stress-related genes. Plant Cell Rep 28(10): 1497-1507

E ZG, Zhang YP, Zhou JH, Wang L (2014) Mini review roles of the bZIP gene family in rice. Genet Mol Res 13:3025-3036

Zhang Y, Zhang G, Xia N, Wang X-J, Li-Li H, Zhen-Sheng K (2008) Cloning and characterization of a bZIP transcription factor gene in wheat and its expression in response to stripe rust pathogen infection and abiotic stresses. Physiol Mol Plant P 73:88-94

Zhou L, Ni E, Yang J, Zhou H et al (2013) Rice OsGL1-6 is involved in leaf Cuticular wax accumulation and drought resistance. PLOS One. https://doi. org/10.1371/journal.pone.0065139

Zhu M, Meng X, Cai J, Li G, Dong T, Li Z (2018) Basic leucine zipper transcription factor SIbZIP1 mediates salt and drought stress tolerance in tomato. BMC Plant Biol 18(1):83

Zou M, Guan Y, Ren H, Zhang F, Chen F (2008) A bZIP transcription factor OsABI5 is involved in rice fertility and stress tolerance. Plant Mol biol 66:675-683. https://doi.org/10.1007/s11103-008-9298-4

\section{Publisher's Note}

Springer Nature remains neutral with regard to jurisdictional claims in published maps and institutional affiliations.

\section{Submit your manuscript to a SpringerOpen ${ }^{\circ}$ journal and benefit from:}

- Convenient online submission

- Rigorous peer review

- Open access: articles freely available online

- High visibility within the field

- Retaining the copyright to your article

Submit your next manuscript at $>$ springeropen.com 